\title{
SUPERFIT: a Computer Code to Fit Surface Resistance and Penetration Depth of a Superconductor
}

\author{
G. Ciovati \\ Jefferson Lab
}

\section{Abstract}

This note describes a computer code that can be used to perform a mean-square fit of experimental data of surface resistance and penetration depth vs. temperature for a superconductor. As a working example, the results from the measurement of a CEBAF niobium single cell cavity will be analysed with this code.

\section{The code}

The computer code has been written with Microsoft Visual $\mathrm{C}++6.0^{\circ}$, which allows the realization of applications in Windows ${ }^{\circledR}$ environment, with an easy user interface.

The surface resistance vs. temperature data can be fitted according to a quasi-exponential formula that provides a simplified version of the BCS theory, valid for temperatures lower than half of the superconductor's critical temperature or using the full BCS theory with the code originally written by J. Halbritter [1] and translated in C++ by J. Yan.

The quasi-exponential formula is:

$$
R_{S}(T)=\frac{a_{0}}{T} \cdot e^{-a_{1} / T}+a_{2}
$$

$a_{2}$ corresponds to the so-called "residual resistance", while $a_{1}$ corresponds to the ratio between the energy gap at zero Kelvin and the Boltzmann's constant.

The penetration depth vs. temperature data can be fitted according to the Two-Fluid model with Pippard correction for the mean free path [2] or using the full BCS theory. For both surface resistance and penetration depth, the BCS theory calculations are done using a boundary condition of diffuse reflection for the electrons, since it represents a situation closer to the real surface of a superconducting cavity.

The formula that describes the corrected Two-Fluid model is:

$$
\lambda(T)=\lambda_{L} \cdot \sqrt{1+\frac{\xi}{l}} \cdot \frac{1}{\sqrt{1-\left(\frac{T}{T_{C}}\right)^{4}}}-\lambda\left(T_{0}\right)
$$

$\lambda_{L}$ is the London penetration depth, $l$ is the mean free path, $\xi$ is the coherence length, $T_{C}$ is the critical temperature and $\lambda\left(T_{0}\right)$ is an additive constant.

The algorithm that performs the mean square fit of the data is the Levenberg-Malquardt algorithm [3], which can be applied to any non-linear fit. It requires the derivative of the function to be fitted with respect to each parameter and, in case of an analytic function (as the quasi-exponential formula for the surface resistance or the Two-Fluid model for the penetration depth), these derivatives can be calculated analytically but when the data have to be fitted with the BCS theory, they have to be computed numerically:

$$
\frac{d y}{d a_{i}}\left(a_{0}, a_{1}, \ldots a_{N}, T_{j}\right)=\frac{y\left(a_{0}, a_{1}, \ldots, a_{i}+h_{i}, \ldots a_{N}\right)-y\left(a_{0}, a_{1}, \ldots, a_{i}, \ldots a_{N}\right)}{h_{i}} \quad i=0, \ldots, N \quad j=0, \ldots, N P
$$


for each parameter $a_{i}$ and temperature value $T_{j}$. The increment $h$ has been chosen to be $5 \%$ of the parameter's value.

As for any non-linear fit, the initial guess value for the parameters is very important to improve the goodness of fit, since initial values too far from the solution may result in an higher chi-squared or a non-convergent calculation. In general, it's a good idea to run the program with initial values equal to the ones resulted from a first fit of the same data.

There exists a version of the code that runs on a DOS shell and, if compiled with a Linux C++ compiler, can run on a Linux machine as well. The Windows ${ }^{\odot}$ version has been called WinSuperfit.

\section{Superfit manual and example}

This section describes the feature of the Windows version of the program.

When the program is launched, two warning messages appear, regarding a library that has been used in the program and is not registered. Just click the "OK" button after few seconds.

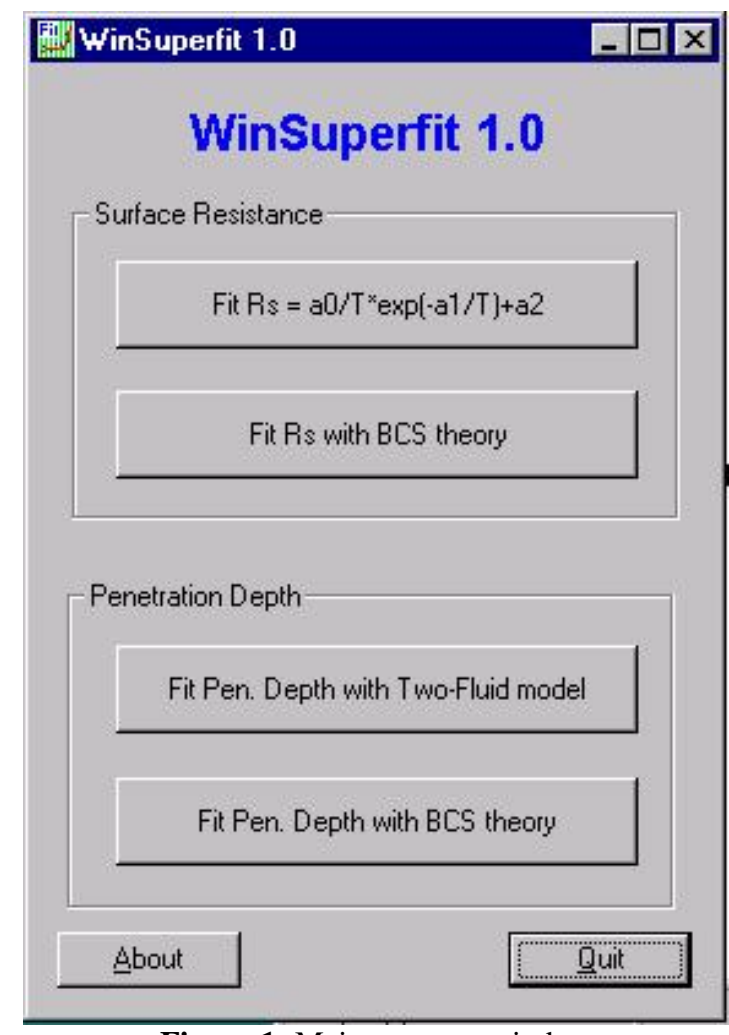

Figure 1: Main program window

The main window shows the four choices to fit surface resistance or penetration depth vs. temperature data. Just click the option you would like to run. Depending on the selected option, a new window appears. On the left there are all the required parameters and a box to be checked if the parameter can be changed during the fit. The default parameters values correspond to niobium. 


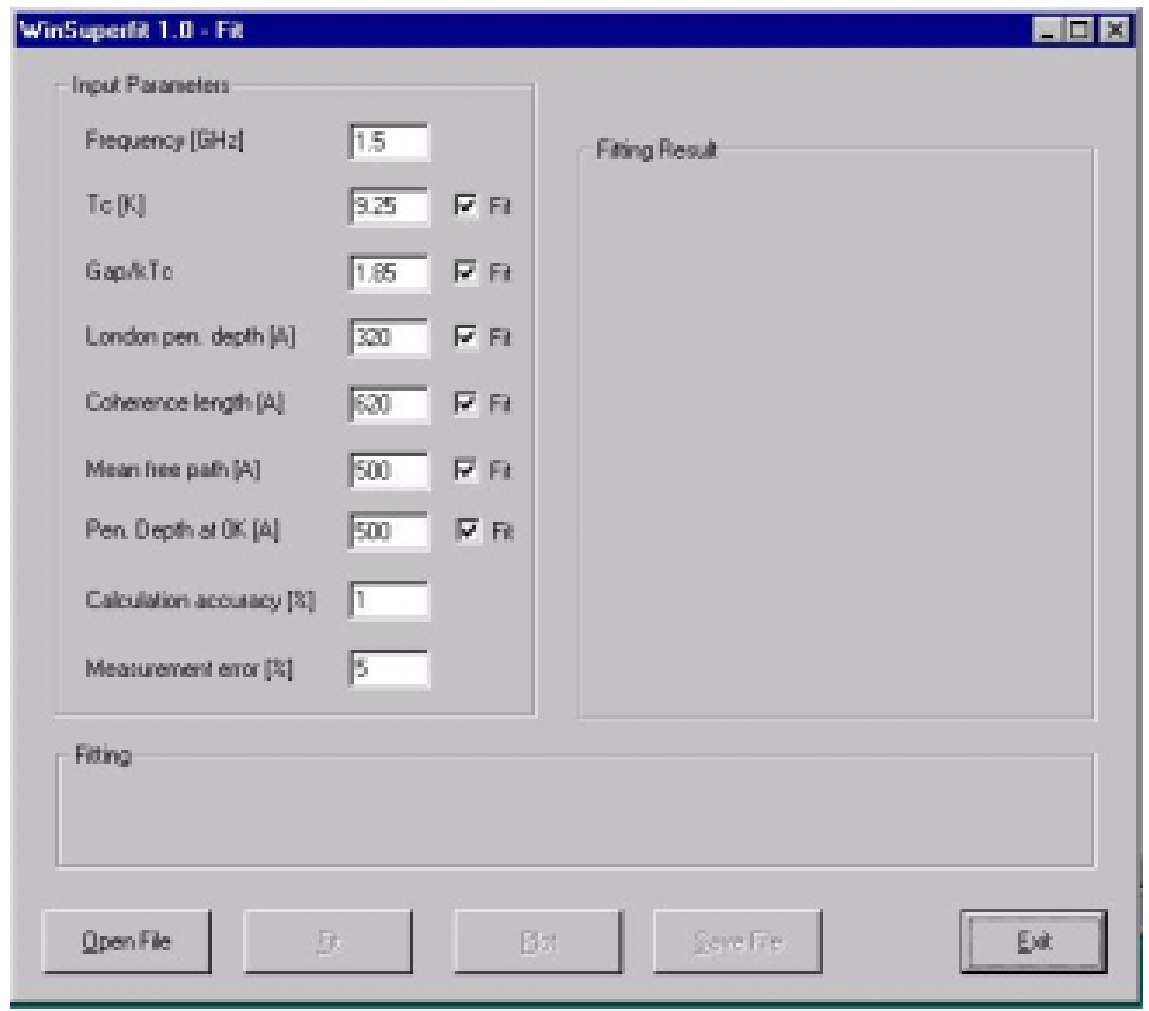

Figure 2: Window for fitting penetration deoth vs. temperature data with BCS theory.

The input data are loaded by pressing the "Open File" button and selecting the filename. It has to be an ASCII file (for example a text file) that begins with the number of data points followed by two or three columns of data, the first one being the temperature values in Kelvin, the second one being the surface resistance (in ohm) or penetration depth (in Angstrom) data. The third column is facultative and contains the experimental error for each data point. The input file can be easily created with Excel. The maximum number of data points is 500 .

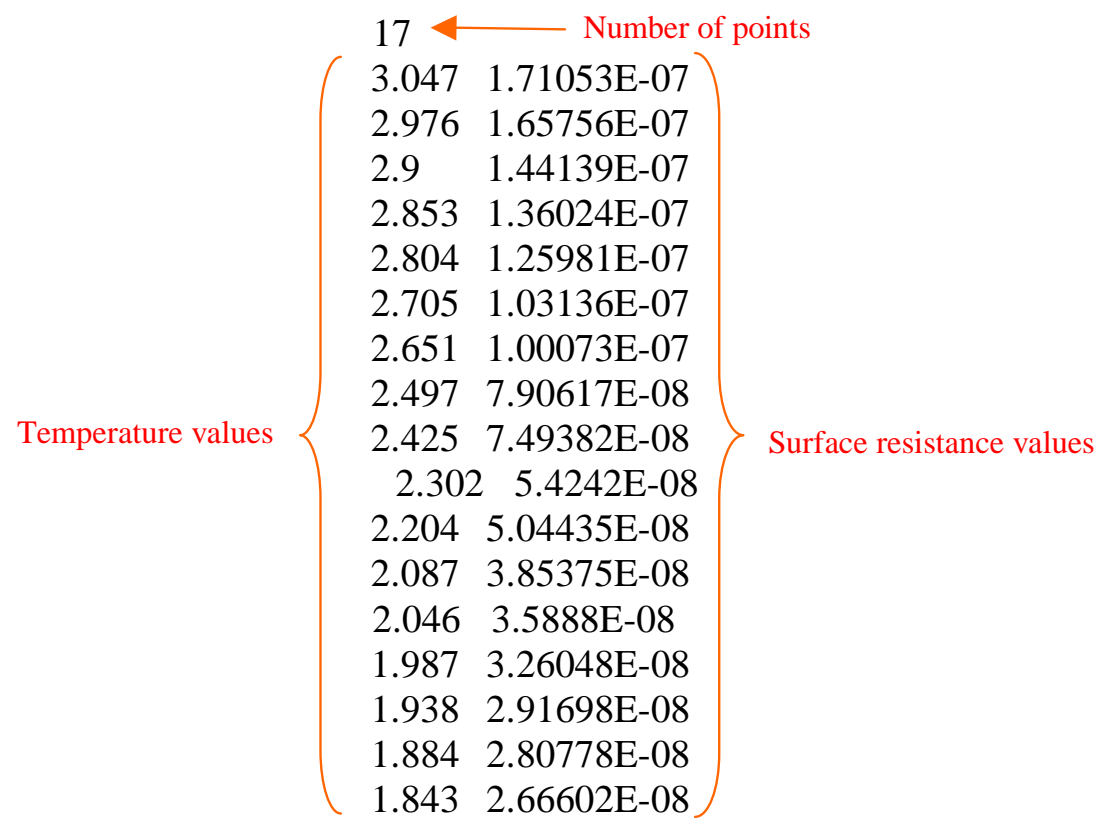

Figure 3: Example of input file 
The program then asks if the measurement errors (third column) is included or not in the input file. In case it is, the "Measurement error" field is disabled; otherwise one can set a percentage value for it. The "Calculation accuracy" field refers to the accuracy with which the BCS theory calculations need to be done.

Once the data are loaded, a "guess" value for the parameters has been assigned and it has been decided which parameters to fit for, the "Fit" button becomes enabled. By pressing it the meansquare fit algorithm starts. The results are shown on the right side of the window.

Fitting data using the BCS theory takes a lot of time and CPU capability, and the results from each iteration step are shown at the bottom of the window. These include the iteration number, the chisquare, the parameter "alamda", which is an indication of the variation applied to the fitting parameters, and finally the fitting parameters $a_{i}(\mathrm{i}=0,1 \ldots 5)$ corresponding to the input parameters listed from top to bottom. The calculations can be stopped at any time by pressing the "Exit" button and then the "Quit" button in the main window.

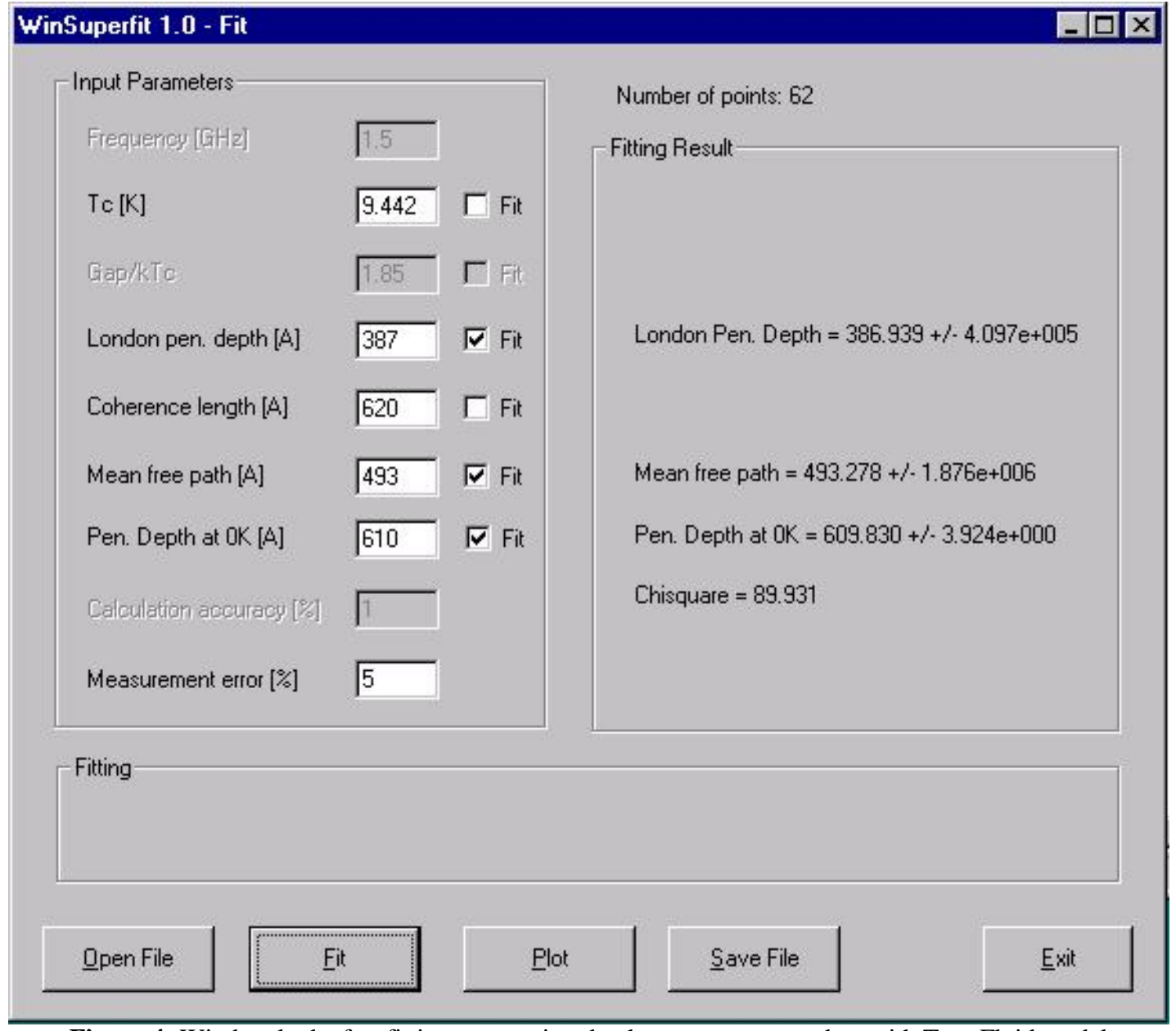

Figure 4: Window look after fitting penetration depth vs. temperature data with Two-Fluid model

Once the fitting process is completed, the "Plot" button is enabled and allows creating a plot of the input data along with a curve describing the fitted function. The plot function has been done using a graphic library included in the book in ref. [4]. 
Data points and fitted function

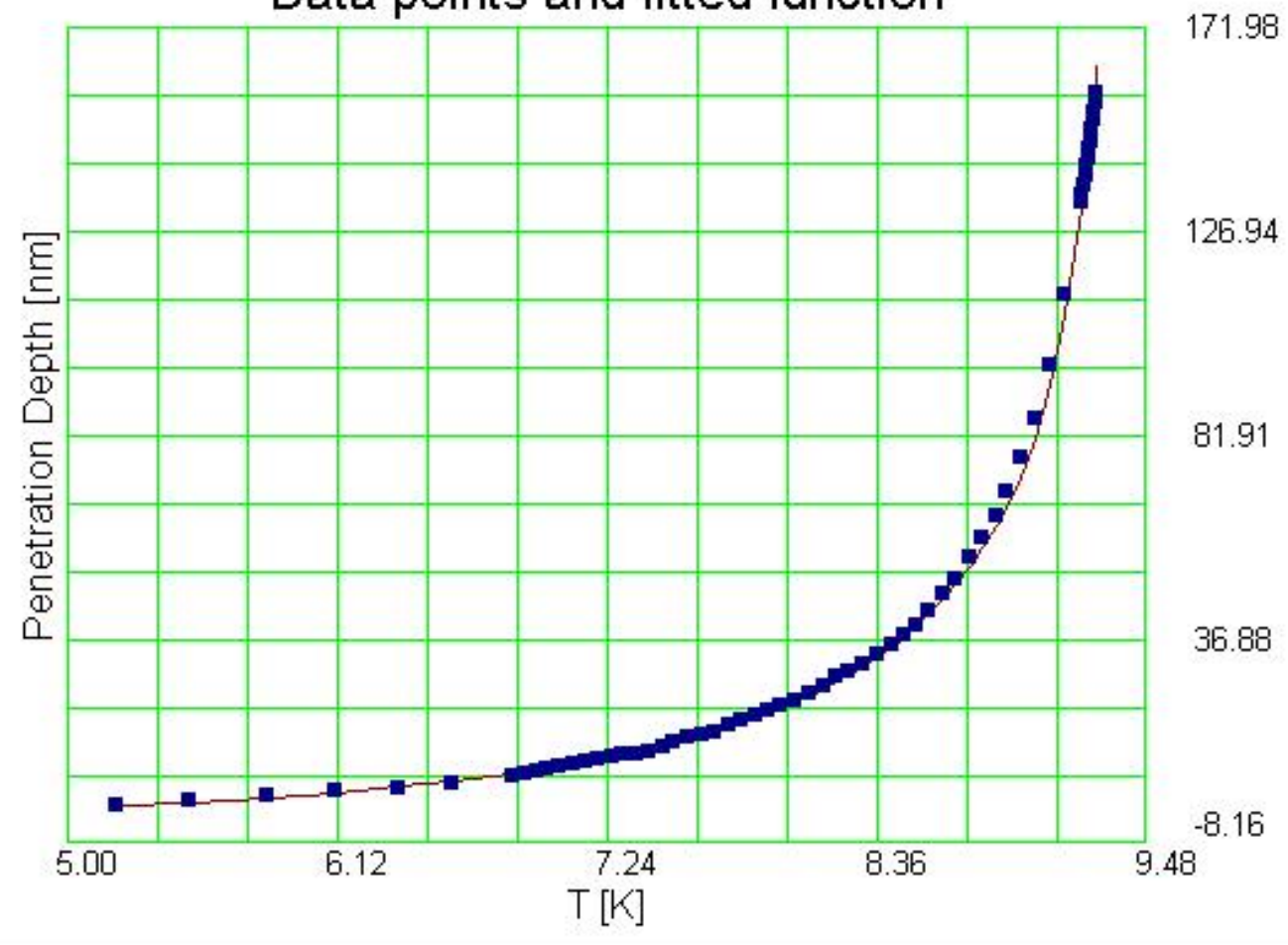

Figure 5: Window that shows input data and fitted function

Pressing the "Save File" button, it is possible to save the initial parameters values, the values after the fit with the chi-square, the input data along with the data obtained from the fitted function and finally the experimental errors.

For example, in figure 6 are shown the results from a fit of the variation of penetration depth with temperature on a CEBAF niobium single cell cavity using the Two-Fluid model. The parameters that could be adjusted during the fit had been chosen to be the critical temperature, the mean free path, the London penetration depth and the additive constant.

Result from fitting Penetration Depth vs. T with two-fluid model

Initial parameters:

Critical temperature (a1) $[\mathrm{K}]=9.442$

London penetration depth (a2) $[\mathrm{A}]=387$

Coherence length (a3) $[\mathrm{A}]=620$

Mean free path (a4) $[A]=493$

Penetration depth at $0 \mathrm{~K}$ (a5) [A] $=610$

chi-squared $=89.9298$

a $1=9.44219+/-0.00553882$

$\mathrm{a} 2=386.993+/-400186$

$\mathrm{a} 4=493.032+/-1.83054 \mathrm{e}+006$

a5 $=609.997+/-6.25004$

$\mathrm{T}[\mathrm{K}]$ Lambda [A] Lambda fit [A] stdev [A]

$\begin{array}{llll}5.205 & 0.321 & 0.323 & 0.016\end{array}$

$\begin{array}{llll}5.510 & 11.543 & 8.423 & 0.577\end{array}$


T [K] Lambda [A] Lambda fit [A] stdev [A]

$\begin{array}{rrrr}5.830 & 24.048 & 18.962 & 1.202 \\ 6.120 & 33.988 & 30.747 & 1.699 \\ 6.375 & 41.683 & 43.284 & 2.084 \\ 6.605 & 48.096 & 56.738 & 2.405 \\ 6.850 & 67.013 & 73.834 & 3.351 \\ 6.900 & 72.785 & 77.733 & 3.639 \\ 6.950 & 77.274 & 81.789 & 3.864 \\ 7.000 & 83.686 & 86.007 & 4.184 \\ 7.050 & 88.496 & 90.398 & 4.425 \\ 7.105 & 92.023 & 95.438 & 4.601 \\ 7.160 & 96.512 & 100.710 & 4.826 \\ 7.210 & 103.245 & 105.718 & 5.162 \\ 7.265 & 108.055 & 111.476 & 5.403 \\ 7.310 & 111.582 & 116.393 & 5.579 \\ 7.370 & 116.392 & 123.257 & 5.820 \\ 7.420 & 121.522 & 129.263 & 6.076 \\ 7.475 & 130.820 & 136.190 & 6.541 \\ 7.525 & 140.760 & 142.799 & 7.038 \\ 7.580 & 150.700 & 150.437 & 7.535 \\ 7.640 & 158.395 & 159.241 & 7.920 \\ 7.695 & 163.846 & 167.779 & 8.192 \\ 7.750 & 176.030 & 176.804 & 8.802 \\ 7.805 & 190.138 & 186.355 & 9.507 \\ 7.860 & 202.322 & 196.477 & 10.116 \\ 7.910 & 208.735 & 206.220 & 10.437 \\ 7.965 & 219.316 & 217.584 & 10.966 \\ 8.025 & 232.142 & 230.830 & 11.607 \\ 8.085 & 249.777 & 245.056 & 12.489 \\ 8.145 & 265.809 & 260.376 & 13.290 \\ 8.195 & 283.444 & 274.072 & 14.172 \\ 8.250 & 298.514 & 290.228 & 14.926 \\ 8.305 & 314.866 & 307.657 & 15.743 \\ 8.365 & 331.539 & 328.315 & 16.577 \\ 8.425 & 353.663 & 350.933 & 17.683 \\ 8.475 & 376.749 & 371.496 & 18.837 \\ 8.530 & 400.797 & 396.186 & 20.040 \\ 8.585 & 430.296 & 423.380 & 21.515 \\ 8.645 & 467.490 & 456.405 & 23.374 \\ 8.695 & 502.439 & 487.090 & 25.122 \\ 8.755 & 546.046 & 528.483 & 27.302 \\ 8.805 & 589.653 & 567.573 & 29.483 \\ 8.860 & 639.672 & 616.520 & 31.984 \\ 8.905 & 695.463 & 662.262 & 34.773 \\ 8.960 & 770.813 & 726.961 & 38.541 \\ 9.020 & 854.179 & 811.964 & 42.709 \\ 9.085 & 970.250 & 927.929 & 48.512 \\ 9.220 & 1125.759 & 1081.605 & 56.288 \\ 9.225 & 137.040 .083 & 1319.053 & 66.452 \\ & & 1319.053 & 67.430 \\ 9.340 .354 & 68.504\end{array}$


$\mathrm{T}$ [K] Lambda [A] Lambda fit [A] stdev [A]

$\begin{array}{llll}9.235 & 1388.041 & 1385.275 & 69.402\end{array}$

$\begin{array}{llll}9.240 & 1409.844 & 1408.991 & 70.492\end{array}$

$\begin{array}{llll}9.245 & 1429.403 & 1433.613 & 71.470\end{array}$

$\begin{array}{llll}9.250 & 1447.038 & 1459.201 & 72.352\end{array}$

$\begin{array}{llll}9.255 & 1466.277 & 1485.819 & 73.314\end{array}$

$\begin{array}{llll}9.260 & 1489.363 & 1513.536 & 74.468\end{array}$

$\begin{array}{llll}9.265 & 1511.166 & 1542.432 & 75.558\end{array}$

$\begin{array}{llll}9.270 & 1530.084 & 1572.589 & 76.504\end{array}$

$\begin{array}{llll}9.275 & 1550.284 & 1604.103 & 77.514\end{array}$

$\begin{array}{llll}9.280 & 1570.484 & 1637.078 & 78.524\end{array}$

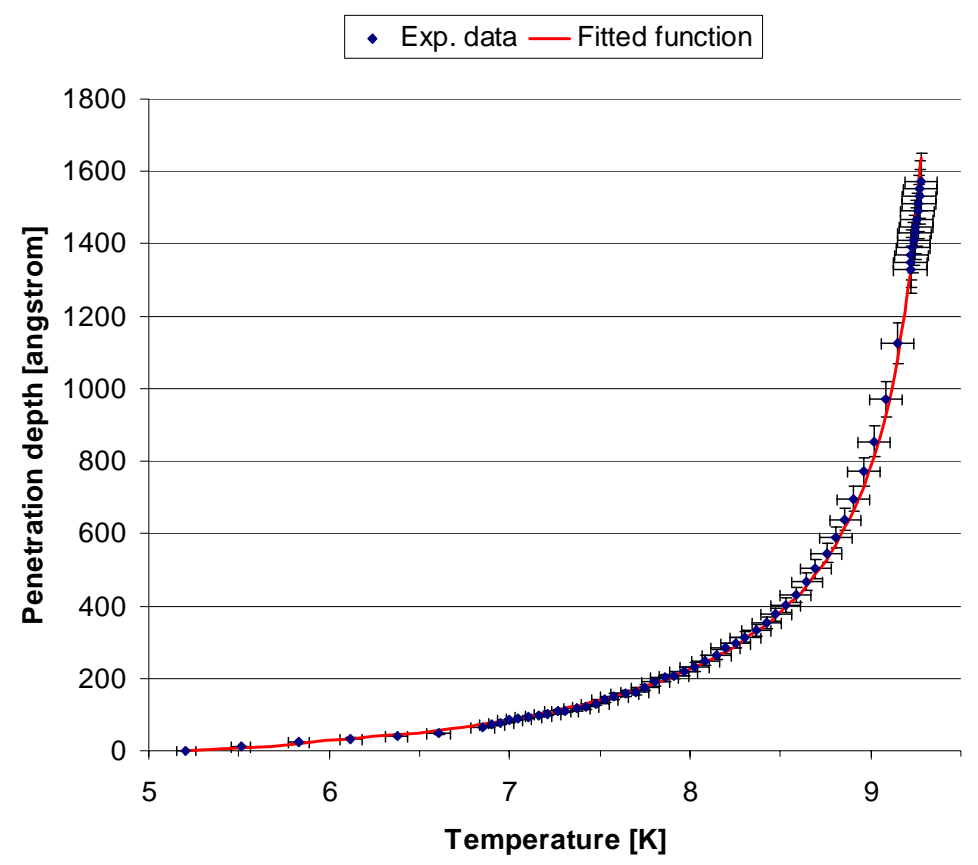

Figure 6: Example of fit of penetration depth vs. temperature.

It can be seen that the estimated error of London penetration depth and mean free path are quite large. This is due to the nature of formula (2), and a more realistic error can be obtained by fitting separately for the two parameters.

The computer code can be requested by sending an e-mail to gciovati@jlab.org.

\section{Acknowledgements}

The author would like to acknowledge J. Yan for translating the BCS theory code in $\mathrm{C}++$.

\section{References}

[1] J. Halbritter, "FORTRAN Program for the computation of the surface impedance of superconductors", KFK-Extern 3/70-6, Karlsruhe, 1970

[2] A. B. Pippard, "An experimental and theoretical study of the relation between magnetic field and current in a superconductor", Proc. of Royal Society, A216, 547 (1953)

[3] W. H. Press, S. A. Teukolsky, W. T. Vetterling, B. P. Flannery, "Numerical Recipes in C++: the art of scientific computing", Cambridge Univ. Press, $2^{\text {nd }}$ ed., 2002

[4] G. Buzzi Ferraris, "Microsoft Visual C++ Applicazioni scientifiche", Mondadori Informatica, 2000, Italy 